\title{
Experimental demonstration of a hyper-entangled ten-qubit Schrödinger cat state
}

\author{
Wei-Bo Gao ${ }^{1}$, Chao-Yang Lu', Xing-Can Yao', Ping Xu', Otfried Gühne ${ }^{2,3}$, Alexander Goebel ${ }^{4}$, \\ Yu-Ao Chen ${ }^{1,4}$, Cheng-Zhi Peng ${ }^{1}$, Zeng-Bing Chen ${ }^{1 \star}$ and Jian-Wei Pan ${ }^{1,4 \star}$
}

\begin{abstract}
Coherent manipulation of a large number of qubits and the generation of entangled states between them has been an important goal and benchmark in quantum information science, leading to various applications such as measurement-based quantum computing ${ }^{1}$ and high-precision quantum metrology ${ }^{2}$. However, the experimental preparation of multiparticle entanglement remains challenging. Using atoms $\mathrm{s}^{3,4}$, entangled states of up to eight qubits have been created, and up to six photons ${ }^{5}$ have been entangled. Here, by exploiting both the photons' polarization and momentum degrees of freedom, we experimentally generate hyper-entangled six-, eight- and ten-qubit Schrödinger cat states with verified genuine multi-qubit entanglement. We also demonstrate super-resolving phase measurements enhanced by entanglement, with a precision to beat the standard quantum limit. Modifications of the experimental set-up would enable the generation of other graph states up to ten qubits. Our method offers a way of expanding the effective Hilbert space and should provide a versatile test-bed for various quantum applications.
\end{abstract}

Control of single photonic qubits using linear optics has been an appealing approach to implementing quantum computing ${ }^{6}$. Experiments in recent years have demonstrated the photons' extremely long decoherence time ${ }^{7}$, fast clock speed ${ }^{8}$, a series of controlled quantum logic gates ${ }^{9,10}$ and algorithms $s^{8,11,12}$ and the generation of multiqubit entangled states ${ }^{5}$. A significant challenge, however, lies in making experimentally accessible sources of photonic multi-qubit states. This is because, on the one hand, the probabilistic nature of spontaneous parametric down-conversion ${ }^{13}$ represents a bottleneck with regard to the attainable brightness and fidelity of multiphoton states based on it: manipulating seven photons or more seems an insurmountable challenge with present technology. On the other hand, triggered single-photon sources from independent quantum dots or other emitters still suffer from spectral and temporal distinguishability, which prevents up-scaling.

There is, however, a way to experimentally control more effectively qubits, by exploiting hyper-entanglement ${ }^{14}$ - the simultaneous entanglement in the multiple degrees of freedom that naturally exist for various physical systems. For instance, one can encode quantum information not only in the polarization of a single photon, but also in its spatial modes ${ }^{15}$, arrival time ${ }^{15}$ or orbital angular momentum ${ }^{16}$. Recently, hyper-entangled photonic states ${ }^{15}$ have been experimentally realized, and shown to offer significant advantages in quantum super-dense coding ${ }^{17,18}$, enhanced violation of local realism ${ }^{19}$, efficient construction of cluster states ${ }^{20,21}$ and multiqubit logic gates ${ }^{10}$.
Although the largest hyper-entangled state ${ }^{15}$ realized so far has expanded the Hilbert space up to 144 dimensions, it is a product state of two-party entangled states and does not involve multipartite entanglement. Other schemes ${ }^{20,21}$ for creating hyperentanglement have been limited by the technical problem of photonic subwavelength phase stability and seem infeasible to generate larger states than the two-photon four-qubit ones. Here, we will describe a method that overcomes these limitations, and the experimental generation of hyper-entangled six-, eight- and ten-qubit photonic Schrödinger cat states.

The Schrödinger cat states, also technically known as Greenberger-Horne-Zeilinger states ${ }^{22}$, involve an equal superposition of two maximally different quantum states. They are of particular interest in quantum mechanics and find wide applications in quantum information processing. Our experiment aims to create the cat state in the form:

$$
\mid \text { Cat }\rangle^{2 n}=\left(|H\rangle^{\otimes n}\left|H^{\prime}\right\rangle^{\otimes n}+|V\rangle^{\otimes n}\left|V^{\prime}\right\rangle^{\otimes n}\right) / \sqrt{2}
$$

where $H$ and $V$ denote horizontal and vertical polarization and $H^{\prime}$ and $V^{\prime}$ label two orthogonal spatial modes (or momentums) of the photons. The state (1) shows maximal entanglement between all photons' polarization and spatial qubits.

Our first experimental step is to generate polarization-entangled $n$-qubit cat states $\mid$ Cat $\rangle_{p}^{n}=\left(|H\rangle^{\otimes n}+|V\rangle^{\otimes n}\right) / \sqrt{2}$. Two pairs of entangled photons are produced by spontaneous parametric downconversion $^{13}$ in the state $(|H\rangle|H\rangle+|V\rangle|V\rangle) / \sqrt{2}$ in paths 2-3 and $4-5$, and a pseudo-single photon source ${ }^{23}$ is prepared in the polarization state $(|H\rangle+|V\rangle) / \sqrt{2}$ in path 1 (see Fig. 1a and the Methods section). Photons 3 and 4 are superposed on a polarizing beam splitter $\left(\mathrm{PBS}_{1}\right)$, and then are further combined with photon 1 on $\mathrm{PBS}_{2}$. Fine adjustments of the delay between the different paths are made to ensure that the photons arrive at the PBSs simultaneously. Furthermore, the photons are spectrally filtered and detected by single-mode fibre-coupled single-photon detectors for good spatial and temporal overlap. As the PBSs transmit $H$ and reflect $V$ polarization, it can be concluded that a coincidence detection of the five output photons implies that all of the photons are either $H$ or $V$ polarized-the two cases are quantum mechanically indistinguishable - therefore projecting them in the cat state $\mid$ Cat $\rangle_{p}^{5}=\left(|H\rangle^{\otimes 5}+|V\rangle^{\otimes 5}\right) / \sqrt{2}$. It is easy to check that, in a similar way, if we combine only photons 1 and 4 (3 and 4$)$ on $\mathrm{PBS}_{2}\left(\mathrm{PBS}_{1}\right)$, entangled cat states between the three photons $1-4-5$ (the four photons 2-3-4-5) can be created by post-selection.

\footnotetext{
${ }^{1}$ Hefei National Laboratory for Physical Sciences at Microscale and Department of Modern Physics, University of Science and Technology of China, Hefei, Anhui 230026, China, ${ }^{2}$ Institut für Quantenoptik und Quanteninformation, Österreichische Akademie der Wissenschaften, Technikerstraße 21A, A-6020 Innsbruck, Austria, ${ }^{3}$ Institut für Theoretische Physik, Universität Innsbruck, Technikerstraße 25, A-6020 Innsbruck, Austria, ${ }^{4}$ Physikalisches Institut, Universität Heidelberg, Philosophenweg 12, D-69120 Heidelberg, Germany. *e-mail: zbchen@ustc.edu.cn; pan@ustc.edu.cn.
} 
a

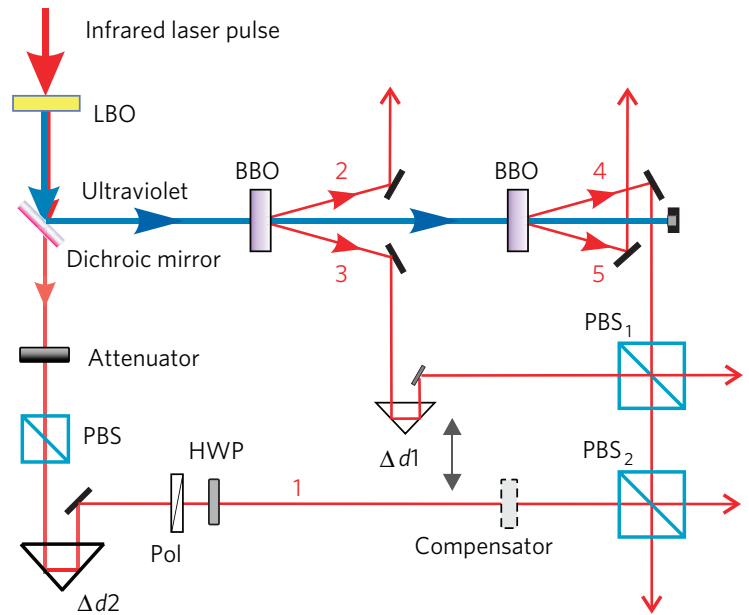

b
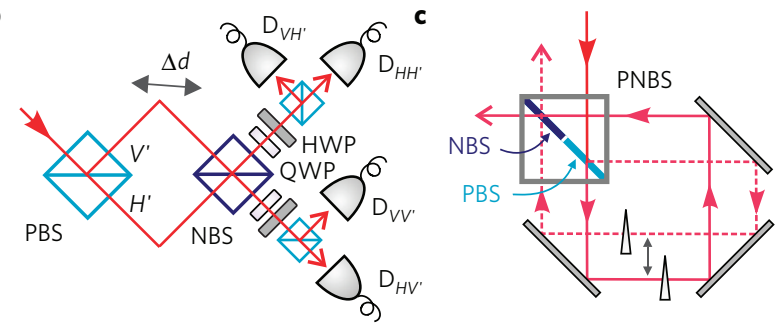

Figure 1 | Experimental set-up for the generation of hyper-entangled sixeight- and ten-qubit Schrödinger cat states. a, A mode-locked Ti:sapphire laser outputs an infrared light pulse with a central wavelength of $788 \mathrm{~nm}$, a pulse duration of $120 \mathrm{fs}$ and a repetition rate of $76 \mathrm{MHz}$, which passes through a $\mathrm{LiB}_{3} \mathrm{O}_{5}(\mathrm{LBO})$ crystal and is converted to ultraviolet light $(\lambda=394 \mathrm{~nm}$ ). Behind the LBO, five dichroic mirrors (only one shown) are used to separate the mixed ultraviolet and infrared light components. The ultraviolet light is focused on two $\beta$-barium borate (BBO) crystals to produce two pairs of entangled photons. The transmitted infrared laser is attenuated to a weak coherent photon source. After the photons are overlapped on the two PBSs, they are guided out to the set-up shown in c. Finally they are detected by fibre-coupled single-photon detectors and the coincidence events are registered by a programmable multichannel coincidence unit. $\mathbf{b}$, Conceptual interferometer for implementation and analysis of hyper-entanglement. An incoming photon is split into two possible spatial modes by the PBS with regard to its polarization: $H \rightarrow H^{\prime}$, $V \rightarrow V^{\prime}$, forming an Einstein-Podolsky-Rosen-like entangled state between the photons' spatial and polarization degrees of freedom. A

Mach-Zehnder-type interferometer with an NBS is used to coherently measure the spatial mode qubit, and subsequently at both of its output ports conventional polarization analysis is carried out. QWP: quarter-wave plate; HWP: half-wave plate. c, The experimentally stable interferometer with a Sagnac-like configuration. The specially designed beam splitter cube (PNBS) is half-PBS coated and half-NBS coated. High-precision small-angle prisms are inserted for fine adjustments of the relative delay of the two different paths.

Next, we grow the polarization-encoded $n$-qubit states into double-sized $2 n$-qubit cat states by planting spatial modes on them. This again exploits the PBSs. Consider a polarized singlephoton qubit in the state $\alpha|H\rangle+\beta|V\rangle$ that passes through a PBS (see Fig. 1b). The PBS separates the photon into two possible spatial modes $H^{\prime}$ and $V^{\prime}$, according to their polarization $H$ and $V$ respectively; indeed, this forms the basis of the PBS as an instrument for measuring polarization. The state of this single photon can now be written as $\alpha|H\rangle\left|H^{\prime}\right\rangle+\beta|V\rangle\left|V^{\prime}\right\rangle$, an entangled state between its polarization and spatial degree of freedom. It is straightforward to extend this method to the $n$-photon state $\mid$ Cat $\rangle_{p}^{n}$; thereby, the hyper-entangled $2 n$-qubit cat state (1) can be created. Besides the cat states, we note that this method can also be flexibly modified for the generation of other graph states ${ }^{24}$, which are central resources in measurement-based quantum computing (see Supplementary Fig. S1).

With multiple degrees of freedom carrying the quantum information in a single photon, measurements of the composite quantum states now become a bit trickier, as it is necessary to read out one degree of freedom without disturbing another one. Figure $1 \mathrm{~b}$ illustrates the apparatus for simultaneously yet independently measuring both the polarization and spatial qubits on the basis of $|0\rangle /|1\rangle$ and $\left(|0\rangle \pm \mathrm{e}^{i \theta}|1\rangle\right)$ (here we denote $|H\rangle=\left|H^{\prime}\right\rangle$ as logic $|0\rangle$ and $|V\rangle=\left|V^{\prime}\right\rangle$ as $\left.|1\rangle\right)$. Specifically, the measurement of the spatial qubit uses an optical interferometer combing the two paths onto a non-polarizing beam splitter (NBS) with an adjustable delay between these two paths that controls the relative phase $\theta$. After this interferometer, the polarization information is then read out by using a combination of a quarter-wave plate, a half-wave plate and a PBS in front of the single-photon detectors, projecting the polarization states into $\left(|H\rangle \pm \mathrm{e}^{i \theta}|V\rangle\right)$. Experimentally, however, it is difficult to directly implement the interferometer in Fig. 1b, because it is sensitive to path length instability of the order of the photon's wavelength. To overcome this problem, we construct intrinsically stable Sagnac-like interferometers with beam-splitter cubes that are half PBS-coated and half NBS-coated (see Fig. 1c). The long-term stabilities and the high visibilities of the five interferometers constructed in our experiment are shown in Supplementary Fig. S2.

As a step-by-step approach, we begin with the creation of the hyper-entangled six-qubit cat state $\mid$ Cat $\rangle^{6}$ and eight-qubit cat state $|\mathrm{Cat}\rangle^{8}$. To analyse the experimentally produced states, we first look at the measurement results in the $|0\rangle /|1\rangle$ basis, as shown in Fig. 2a,b for $\mid$ Cat $\rangle^{6}$ and $\mid$ Cat $\rangle^{8}$, respectively. For ideal cat states, the desired combinations in this basis should in principle be only $|H\rangle^{\otimes n}\left|H^{\prime}\right\rangle^{\otimes n}$ and $|V\rangle^{\otimes n}\left|V^{\prime}\right\rangle^{\otimes n}$. This is confirmed by the experimental data in Fig. 2a,b, showing that these two terms dominate the overall coincidence events, with a signal-to-noise ratio (defined as the ratio of the average of the desired components to that of the other non-desired ones) of 85:1 to 1,100:1 for the states $\mid$ Cat $\rangle^{6}$ and $\mid$ Cat $\rangle^{8}$, respectively. We note that the undesired noise, noticeably located in the diagonal line of Fig. 2a,b, mainly arises from the double-pair emission of entangled photons.

Although the above data determine the population in the $|0\rangle /|1\rangle$ basis of the cat states, they are not sufficient to reveal their coherence properties. We took therefore additional measurements in the basis $|R\rangle=\left(|0\rangle+\mathrm{e}^{i \theta}|1\rangle\right) / \sqrt{2}$ and $|L\rangle=\left(|0\rangle-\mathrm{e}^{i \theta}|1\rangle\right) / \sqrt{2}$. In this new basis, the cat state $\mid$ Cat $\rangle^{n}$ can be written in the form of $(|R\rangle+|L\rangle)^{\otimes n}+\mathrm{e}^{-i n \theta}(|R\rangle-|L\rangle)^{\otimes n}$; thus, the probabilities of creating the components $|R\rangle^{\otimes n},|R\rangle^{\otimes(n-1)}|L\rangle, \ldots,|L\rangle^{\otimes n}$ and hence the experimentally observed coincidence events should vary as $\sim(1 \pm \cos n \theta)$. From these measurements, one can determine the expectation values of the spin observable: $\left\langle M_{\theta}{ }^{\otimes n}\right\rangle=\cos n \theta$, where $M_{\theta}=\cos \theta \sigma_{x}+\sin \theta \sigma_{y}$, which oscillates $n$ times sinusoidally over a single cycle of $2 \pi$. Indeed, this can arise only from coherent superposition between the $|0\rangle^{\otimes n}$ and $|1\rangle^{\otimes n}$ component of the cat state and serves as a characteristic signature of $n$-qubit coherence ${ }^{3}$.

Figure $2 \mathrm{c}, \mathrm{d}$ shows the experimentally obtained expectation values $\left\langle M_{\theta}{ }^{\otimes n}\right\rangle$ as a function of $\theta(0 \leq \theta \leq 2 \pi)$ and the fitted sinusoidal fringes. The fringes clearly show the $n \theta$ oscillation, with a visibility of $0.527 \pm 0.002$ and $0.67 \pm 0.01$ for the six- and eightqubit cat states, respectively, confirming the coherence between all effective $n$ qubits encoded with either polarization or spatial information. We note that the reduction of the visibilities is caused by, besides the above-mentioned double-pair photon emission, also the imperfections of photon overlapping at the PBSs and NBSs. 
a

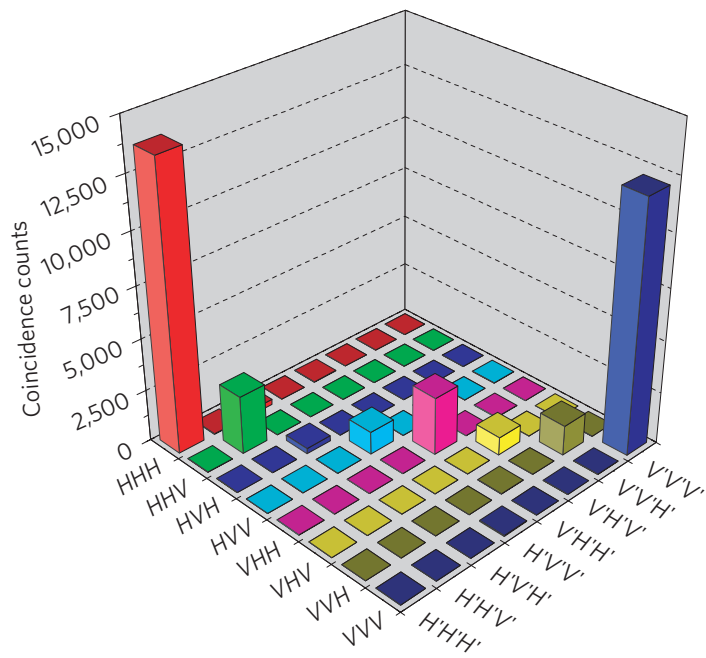

c

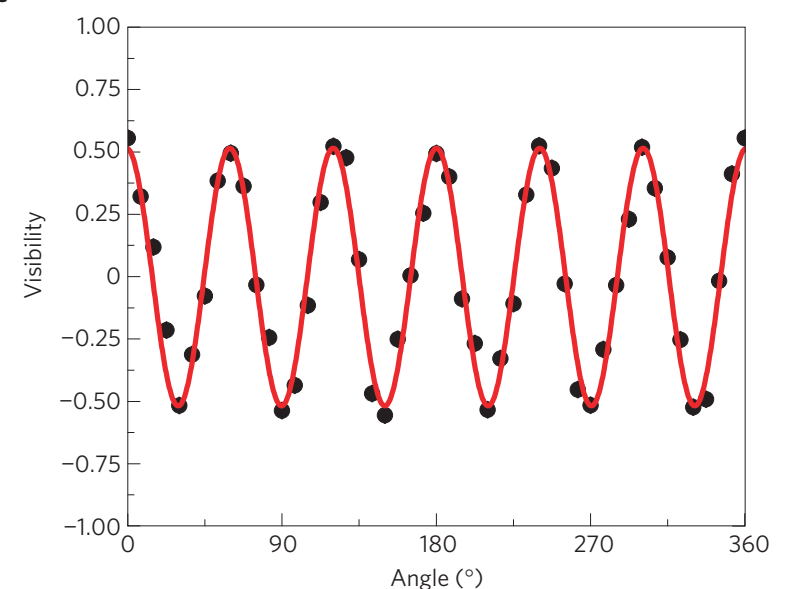

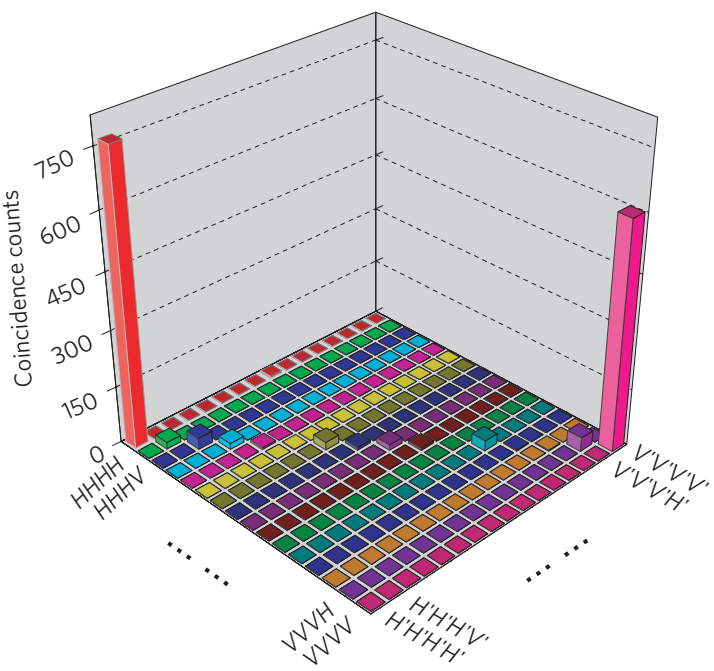

d

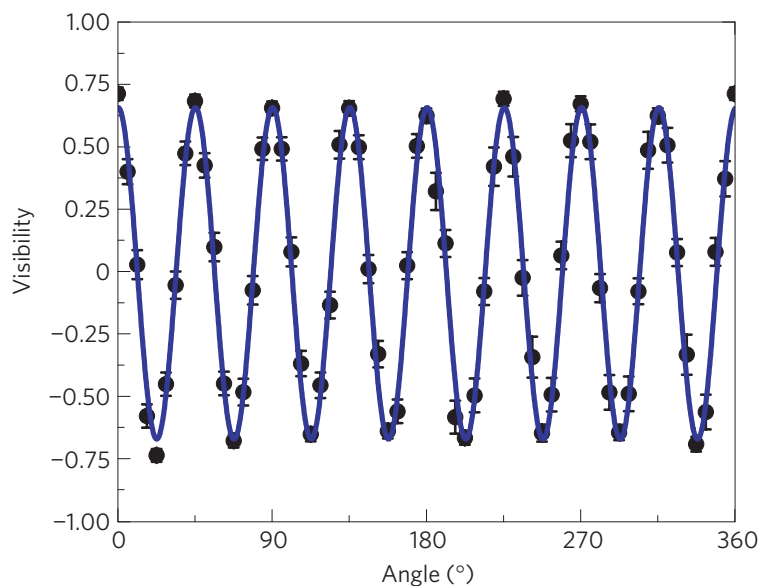

Figure 2 | Experimental results for determination of the fidelities of the six- and eight-qubit cat states, showing phase super-resolution.

a,b, Coincidence counts obtained in the $|H\rangle /|V\rangle\left(\left|H^{\prime}\right\rangle /\left|V^{\prime}\right\rangle\right)$ basis, accumulated for 150 s and 480 s for the six- (a) and eight-qubit (b) state respectively. $\mathbf{c}, \mathbf{d}$, Measurement results obtained along the basis of $\left(|H\rangle+\mathrm{e}^{i \theta}|V\rangle\right) / \sqrt{2}\left(\left(\left|H^{\prime}\right\rangle+\mathrm{e}^{i \theta}\left|V^{\prime}\right\rangle\right) / \sqrt{2}\right)$, showing the coherence of the cat states and phase super-resolution fringes (see text). The error bars represent one standard deviation (in $\mathbf{c}$ they are smaller than the dots representing the measurement points) deduced from propagated Poissonian counting statistics of the raw detection events.

From the data shown in Fig. 2, we can further determine the fidelities of the cat states and detect the presence of genuine multipartite entanglement ${ }^{25}$. The fidelity-a measure of the extent to which the desired state is created-is the overlap of the experimentally produced state with the ideal one: $F(|\psi\rangle)=\left\langle\psi\left|\rho_{\exp }\right| \psi\right\rangle$. For the cat state, $|\psi\rangle\langle\psi|$ can be decomposed as ${ }^{5}(1 / 2)\left[(|0\rangle\langle 0|)^{\otimes n}+\right.$ $\left.(|1\rangle\langle 1|)^{\otimes n}+(1 / n) \sum_{k=1}^{n}(-1)^{k} M_{(k \pi / n)}^{\otimes n}\right]$, corresponding to measurements in the basis of $|0\rangle /|1\rangle$ and $\left(|0\rangle \pm \mathrm{e}^{i(k \pi / n)}|1\rangle\right)$. Figure 2a-d shows the experimental results, from which the fidelities of the six- and eight-qubit cat states can be determined: $F\left(|\mathrm{Cat}\rangle^{6}\right)=$ $0.6308 \pm 0.0015, F\left(|\mathrm{Cat}\rangle^{8}\right)=0.776 \pm 0.006$. The notion of genuine multipartite entanglement characterizes whether generation of the state requires interaction of all parties, distinguishing the experimentally produced state from any incompletely entangled state. For cat states, it is sufficient for the presence of genuine multipartite entanglement that their fidelities exceed the threshold of 0.5 (ref. 25). Thus, with high statistical significance, genuine $n$-qubit entanglement of the cat states created in our experiment is confirmed. We note that the fidelity of the six-qubit cat state is considerably lower than that of the eight-qubit state, which is due to the fact that the generation of the former involves a faint coherent laser light, which introduces more noise than the configuration of the latter. It is worth mentioning here an advantage that the hyper-entanglement brings-our new six-qubit cat state not only has a higher fidelity than the previous six-photon cat state ${ }^{5}$, but also its count rate reaches $\sim 200 \mathrm{~s}^{-1}$, some 4 orders of magnitude brighter than the six-photon coincidence.

The fringes in Fig. $2 \mathrm{c}$,d show $n \theta$ phase dependencies, which are $n$ times more sensitive to phase changes than that of a single qubit, highlighting the potential use of the cat states for super-resolving phase measurements ${ }^{2}$. Suppose we need to estimate the phase $\varphi$ of a process $|0\rangle \rightarrow|0\rangle$ and $|1\rangle \rightarrow \mathrm{e}^{i \varphi}|1\rangle$. A simple initial probe state $\left|\psi_{i}\right\rangle=$ $(|0\rangle+|1\rangle) / \sqrt{2}$ would evolve into $\left|\psi_{f}\right\rangle=\left(|0\rangle+\mathrm{e}^{i \varphi}|1\rangle\right) / \sqrt{2}$ and the phase can be estimated from the overlap $\left|\left\langle\psi_{i} \mid \psi_{f}\right\rangle\right|^{2}=[1+\cos (\varphi)] / 2$. Provided with $N$ probe states, the uncertainty of the phase estimation is $\Delta \varphi=1 / \sqrt{N}$ - the standard quantum limit. Applying the same dynamics on $N$ qubits in a cat state $\mid$ Cat $\rangle^{N}=\left(|0\rangle^{\otimes N}+\right.$ $\left.|1\rangle^{\otimes N}\right) / \sqrt{2}$, it will evolve to $\left|\mathrm{Cat}_{f}\right\rangle^{N}=\left(|0\rangle^{\otimes N}+\mathrm{e}^{i N \varphi}|1\rangle^{\otimes N}\right) / \sqrt{2}$. Then, measuring the overlap or the mean value $\left\langle M_{\varphi}{ }^{\otimes N}\right\rangle$ leads to dependence on $\cos (N \varphi)$, from which the phase can be determined with an error of $\Delta \varphi=1 / N$ (refs $2,3,26$ ). The $1 / N$ scaling of the precision is called the Heisenberg limit, as it coincides with the limit imposed by the uncertainty principle. As shown in Fig. $2 \mathrm{c}, \mathrm{d}$, we have observed the expected $n$-times phase super-resolution for the six- and eight-qubit cat states, with a visibility of $0.527 \pm 0.002$ and $0.67 \pm 0.01$, respectively. These values are larger than the threshold 
a

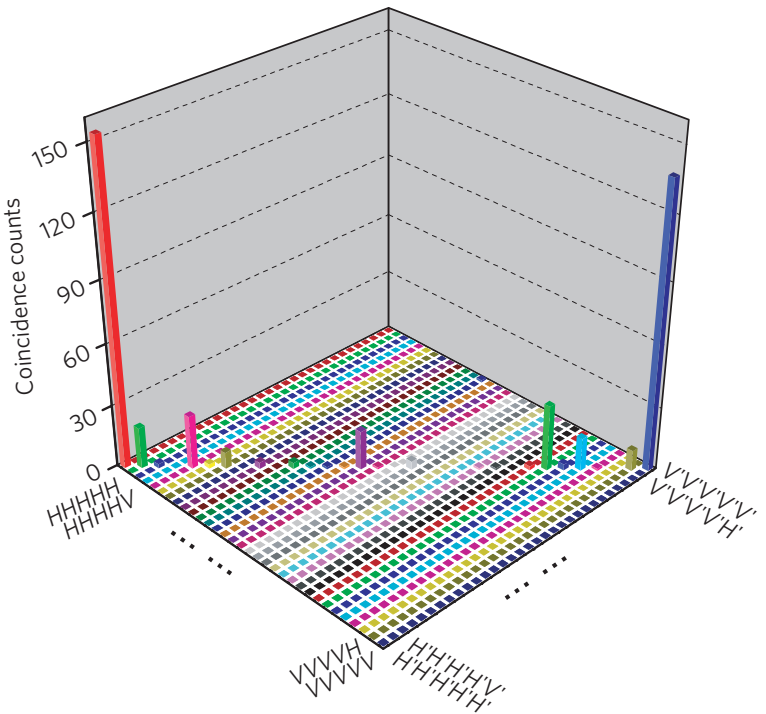

b

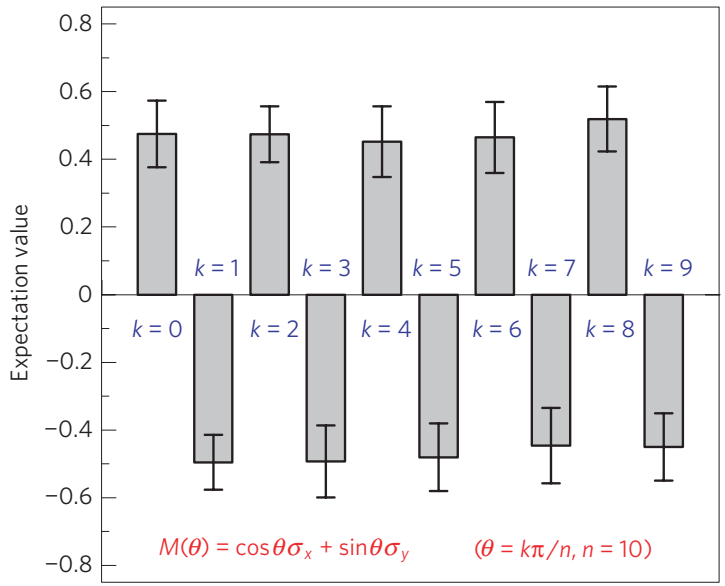

Figure 3 | Experimental results for the ten-qubit cat states. a, Coincidence counts measured in the $|H\rangle /|V\rangle\left(\left|H^{\prime}\right\rangle /\left|V^{\prime}\right\rangle\right)$ basis for $6 \mathrm{~h}$. b, The expectation values of $M_{\theta}{ }^{\otimes 10}$, each derived from a complete set of 1,024 sixfold coincidence events in $1.5 \mathrm{~h}$ in the basis of $|H\rangle \pm \mathrm{e}^{i k \pi / 10}|\mathrm{~V}\rangle\left(\left|H^{\prime}\right\rangle \pm \mathrm{e}^{i k \pi / 10}\left|\mathrm{~V}^{\prime}\right\rangle\right)$. The error bar denotes one standard deviation calculated from Poissonian counting statistics of the detection events.

of $1 / \sqrt{N}$ for phase super-resolution, and taking into account the cat-state preparation efficiency of $1 / 2$ caused by the PBS filtering (the efficiency can be improved to unity in principle ${ }^{6}$ ), we find that the eight-qubit cat state can achieve a phase sensitivity $1.13 \pm 0.02$ times greater than the standard quantum limit (see Supplementary Information). This represents entanglement-enhanced quantum metrology with the largest number of qubits so far. However, as with all photonic experiments ${ }^{27}$, the single-photon detection efficiency needs to be improved significantly for a practical implementation of Heisenberg-limited measurements.

Now we proceed with describing the generation and analysis of the ten-qubit cat state $\mid$ Cat $\rangle^{10}$, which uses the full set-up shown in Fig. 1. As a result of the probabilistic nature of spontaneous parametric down-conversion, the coincidence count rate of the ten-qubit state is as low as $0.021 \mathrm{~Hz}, 1 / 160(1 / 11,000)$ of that of the eight-qubit (six-qubit) state. Measurement results along the $|0\rangle /|1\rangle$ basis are shown in Fig. 3a with all $2^{10}=1,024$ possible combinations $\left(|H\rangle^{\otimes 5}\left|H^{\prime}\right\rangle^{\otimes 5},|H\rangle^{\otimes 5}\left|H^{\prime}\right\rangle^{\otimes 4}\left|V^{\prime}\right\rangle, \ldots,|V\rangle^{\otimes 5}\left|V^{\prime}\right\rangle^{\otimes 5}\right)$ plotted, giving a signal to noise ratio (defined as above) of 940:1. We further take measurements in the $\left(|0\rangle \pm \mathrm{e}^{i \theta}|1\rangle\right)$ basis, where $\theta$ is chosen as: $\theta=k \pi / 10, k=0,1, \ldots, 9$. The measured expectation values of the observable $M_{\theta}{ }^{\otimes 10}$ are listed in Fig. 3b with an average absolute value of 0.475 - this can also be seen equivalently as the fringe visibility shown in Fig. 2c,d. We can thus calculate the state fidelity: $\left.F(\mid \text { Cat }\rangle^{10}\right)=0.561 \pm 0.019$, which is above the threshold of 0.5 by more than three standard deviations, thus establishing the presence of genuine ten-qubit entanglement after post-selection. These data are further analysed using an optimized entanglement witness method (see Supplementary Information), which, with even higher significance, confirms that the entanglement truly involves all ten qubits.

We have experimentally analysed the hyper-entangled six-, eight- and ten-qubit photonic Schrödinger cat states and their phase super-resolution. These results represent the largest entangled state realized so far, expanding the effective Hilbert space up to 1,024 dimensions. The cat states demonstrated here, together with other graph states technically feasible within our experimental method (see Supplementary Fig. S1), create a versatile testing ground for the study of non-locality ${ }^{28}$, multipartite entanglement and many quantum information protocols. Indeed, by taking advantage of the hyper-entanglement, it is now possible to reach some experimental regimes that were hardly accessible before, for instance, demonstrations of the robustness of anyonic braiding ${ }^{29}$ and topological cluster-state encoding ${ }^{30}$ that require manipulation of 7-10 qubits. It will be interesting in future work to further exploit quantum particles' other degrees of freedom, such as arrival time and orbital angular momentum, to create larger multidimensional entangled states, aiming for more efficient quantum information processing.

\section{Methods}

The photon pairs and single photons for the later observation of the cat state are generated as follows: as shown in Fig. 1a, a femtosecond infrared laser is attenuated to be a weak coherent photon source that has a very small probability $(p \sim 0.03)$ of containing a single photon for each pulse, and prepared in the superposition state $(|H\rangle+|V\rangle) / \sqrt{2}$ in path 1. Meanwhile, a pulsed ultraviolet (ultraviolet) laser, which is up-converted from the intense infrared laser, passes through two $\beta$-barium borate crystals, generating two pairs of entangled photons in the state $(|H\rangle|H\rangle+|V\rangle|V\rangle) / \sqrt{2}$ in paths $2-3$ and 4-5. The photon pairs have an average two-photon coincidence count rate of $2.4 \times 10^{4} \mathrm{~s}^{-1}$ and a visibility of 0.92 in the $H / V$ basis and 0.90 in the $(H \pm V) / \sqrt{2}$ basis.

Received 16 April 2009; accepted 27 January 2010; published online 14 March 2010

\section{References}

1. Raussendorf, R. \& Briegel, H. J. A one-way quantum computer. Phys. Rev. Lett. 86, 5188-5191 (2001).

2. Giovannetti, V., Lloyd, S. \& Maccone, L. Quantum-enhanced measurements: Beating the standard quantum limit. Science 306, 1330-1336 (2004).

3. Leibfried, D. et al. Creation of a six-atom 'Schrödinger cat' state. Nature 438, 639-642 (2005).

4. Häffner, H. et al. Scalable multiparticle entanglement of trapped ions. Nature 438, 643-646 (2005).

5. Lu, C.-Y. et al. Experimental entanglement of six photons in graph states. Nature Phys. 3, 91-95 (2007).

6. Kok, P. et al. Linear optical quantum computing with photonic qubits. Rev. Mod. Phys. 79, 135-174 (2007).

7. Ursin, R. et al. Entanglement-based quantum communication over $144 \mathrm{~km}$. Nature Phys. 3, 481-486 (2007).

8. Prevedel, R. et al. High-speed linear optics quantum computation using active feed-forward. Nature 445, 65-69 (2007).

9. Politi, A., Cryan, M. J., Rarity, J. G., Yu, S. \& O’Brien, J. L. Silica-on-silicon waveguide quantum circuits. Science 320, 646-649 (2008).

10. Lanyon, B. P. et al. Simplifying quantum logic using higher-dimensional Hilbert spaces. Nature Phys. 5, 134-140 (2009).

11. Tame, M. S. et al. Experimental realization of Deutsch's algorithm in a one-way quantum computer. Phys. Rev. Lett. 98, 140501 (2007). 
12. Lanyon, B.P. et al. Experimental demonstration of a complied version of Shor's algorithm with quantum entanglement. Phys. Rev. Lett. 99, 250505 (2007).

13. Kwiat, P. G. et al. New high-intensity source of polarization-entangled photon pairs. Phys. Rev. Lett. 75, 4337-4341 (1995).

14. Kwiat, P. G. Hyper-entangled states. J. Mod. Opt. 44, 2173-2184 (1997).

15. Barreiro, J. T., Langford, N. K., Peter, N. A. \& Kwiat, P. G. Generation of hyperentangled photon pairs. Phys. Rev. Lett. 95, 260501 (2005).

16. Mair, A., Vaziri, A., Weihs, G. \& Zeilinger, A. Entanglement of the orbital angular momentum states of photons. Nature 412, 313-316 (2001).

17. Schuck, C., Huber, G., Kurtsiefer, C. \& Weinfurter, H. Complete deterministic linear optics Bell state analysis. Phys. Rev. Lett. 96, 190501 (2006).

18. Barreiro, J. T., Wei, T. C. \& Kwiat, P. G. Beating the channel capacity limit for linear photonic superdense coding. Nature Phys. 4, 282-286 (2008).

19. Barbieri, M., Martini, F. D., Mataloni, P., Vallone, G. \& Cabello, A. Enhancing the violation of the Einstein-Podolsky-Rosen local realism by quantum hyperentanglement. Phys. Rev. Lett. 97, 140407 (2006).

20. Chen, K. et al. Experimental realization of one-way quantum computing with two-photon four-qubit cluster states. Phys. Rev. Lett. 99, 120503 (2007).

21. Vallone, G. et al. Active one-way quantum computation with two-photon four-qubit cluster states. Phys. Rev. Lett. 100, 160502 (2008).

22. Greenberger, D. M., Horne, M., Shimony, A. \& Zeilinger, A. Bell's theorem without inequalities. Am. J. Phys. 58, 1131-1143 (1990).

23. Rarity, J. G. \& Tapster, P. R. Three-particle entanglement from entangled photon pairs and a weak coherent state. Phys. Rev. A 59, R35-R38 (1999).

24. Hein, M., Eisert, J. \& Briegel, H. J. Multiparty entanglement in graph states. Phys. Rev. A 69, 062311 (2004).

25. Bourennane, M. et al. Experimental detection of multipartite entanglement using witness operators. Phys. Rev. Lett. 92, 087902 (2004).

26. Leibfried, D. et al. Toward Heisenberg-limited spectroscopy with multiparticle entangled state. Science 304, 1476-1478 (2004).
27. Dowling, J. P. Quantum optical metrology-the lowdown on high-N00N states. Contemp. Phys. 49, 125-143 (2008).

28. Cabello, A. Bipartite Bell inequalities for hyperentangled states. Phys. Rev. Lett. 97, 140406 (2006)

29. Han, Y.-J., Raussendorf, R. \& Duan, L.-M. Scheme for demonstration of fractional statistics of anyons in an exactly solvable model. Phys. Rev. Lett. 98, 150404 (2007).

30. Raussendorf, R., Harrington, J \& Goyal, K. Topological fault-tolerance in cluster state quantum computation. New J. Phys. 9, 199 (2007).

\section{Acknowledgements}

We thank J.P. Dowling and S.J. van Enk for helpful discussions. This work was supported by the National Natural Science Foundation of China, the Chinese Academy of Sciences and the National Fundamental Research Program (under Grant No 2006CB921900). This work was also supported by the Alexander von Humboldt Foundation, the ERC, the FWF (START prize) and the EU (SCALA, OLAQUI, QICS).

\section{Author contributions}

C.-Y.L., W.-B.G. and J.-W.P. conceived the research; W.-B.G., X.-C.Y., P.X., A.G., Y.-A.C. and C.-Z.P. carried out the experiment; O.G. contributed theoretical analytic tools; W.-B.G., O.G. and C.-Y.L. analysed the data; C.-Y.L., O.G., W.-B.G. and J.-W.P. wrote the paper; J.-W.P. and Z.-B.C. supervised the whole project.

\section{Additional information}

The authors declare no competing financial interests. Supplementary information accompanies this paper on www.nature.com/naturephysics. Reprints and permissions information is available online at http://npg.nature.com/reprintsandpermissions. Correspondence and requests for materials should be addressed to Z.-B.C. or J.-W.P. 